\title{
Subjective Rating of Weak Tactile Stimuli Is Parametrically Encoded in Event-Related Potentials
}

\author{
Ryszard Auksztulewicz ${ }^{1}$ and Felix Blankenburg ${ }^{1,2,3}$ \\ ${ }^{1}$ Berlin School of Mind and Brain, Humboldt-Universität zu Berlin, 10099 Berlin, Germany, ${ }^{2}$ Dahlem Institute for Neuroimaging of Emotions, Freie \\ Universität Berlin, 14195 Berlin, Germany, and ${ }^{3}$ Center for Adaptive Rationality, Max Planck Institute for Human Development, 14195 Berlin, Germany
}

Neural signatures of somatosensory awareness have often been studied by examining EEG responses to hardly detectable stimuli. Previous reports consistently showed that event-related potentials (ERPs) measured over early somatosensory cortex diverge for detected and missed perithreshold stimuli at 80-100 ms after stimulus onset. So far, however, all previous studies have operationalized somatosensory awareness as binary stimulus detection.

Here, we investigated whether ERP components attributed to neuronal activity in early somatosensory cortices would parametrically reflect subjective ratings of stimulus awareness. EEG (64 channel) was recorded in human participants $(N=20)$, with perithreshold electrical stimulation applied to the left median nerve. Participants indicated perceptibility on a continuous visual rating scale, and stimulation intensity was readjusted in each block to a perithreshold level. The aim of the analysis was to investigate which brain areas reflect the subsequent perceptual awareness ratings parametrically, and how early such parametric effects occur.

Parametric ERP effects were found as early as $86 \mathrm{~ms}$ after stimulus onset. This parametric modulation of ERP amplitude was source localized to secondary somatosensory cortex, and attributed to feedforward processing between primary and secondary somatosensory cortex by means of dynamic causal modeling (DCM). Furthermore, later in the analysis window, the subjective rating of stimuli correlated with the amplitude of the N140 component and with a broadly distributed P300 component. By DCM modeling, these late effects were explained in terms of recurrent processing within the network of somatosensory and premotor cortices. Our results indicate that early neural activity in the somatosensory cortex can reflect the subjective quality of tactile perception.

\section{Introduction}

Despite a sustained interest in the scientific study of consciousness, the question concerning which neuronal processes constitute conscious perception is far from being resolved (Dehaene and Changeux, 2011). Opinions differ on whether perceptual awareness operates predominantly within the sensory cortices (Zeki, 2001; Lamme, 2006; Tallon-Baudry, 2011), or between sensory areas and frontal regions (Dehaene et al., 2006). The architecture of the somatosensory system, with a large spatial separation of primary and secondary cortices, makes the tactile modality particularly well suited for noninvasive investigation of local cortical interactions related to conscious perception (Romo et al., 2012).

Several previous studies probed the neural signatures of tactile awareness by measuring EEG responses to perithreshold stimuli, typically showing event-related potential (ERP) divergence for

\footnotetext{
Received Sept. 5, 2012; revised May 29, 2013; accepted June 7, 2013.

Author contributions: R.A. and F.B. designed research; R.A. performed research; R.A. and F.B. analyzed data; R.A. and F.B. wrote the paper.

This work was supported by a grant from the German Federal Ministry of Education and Research (Bundesministerium für Bildung und Forschung) to F.B. We thank Timo Schmidt for help with data acquisition, as well as David Kassim, Dar Meshi, Dirk Ostwald, and Bernhard Spitzer for helpful advice.

The authors declare no financial conflicts of interest.

Correspondence should be addressed to Ryszard Auksztulewicz, Berlin School of Mind and Brain, Luisenstrasse

56, Haus 1, 10099 Berlin, Germany. E-mail: ryszard.auksztulewicz@gmail.com.

DOI:10.1523/JNEUROSCI.4243-12.2013

Copyright $\odot 2013$ the authors $\quad 0270-6474 / 13 / 3311878-10 \$ 15.00 / 0$
}

detected and missed stimuli 70-100 ms after stimulus presentation (Schubert et al., 2006; Jones et al., 2007; Auksztulewicz et al., 2012). This effect was ascribed to differential activity in early somatosensory cortex using a biophysically realistic computational model of primary somatosensory cortex (Jones et al., 2007), as well as network analysis using dynamic causal modeling (DCM; Auksztulewicz et al., 2012). The latter study provided evidence that increased recurrent processing between contralateral primary (cSI) and secondary (cSII) somatosensory cortex explains differences in late ERP components between detected and missed tactile stimuli, and thus may be a plausible mechanism underlying somatosensory detection (Auksztulewicz et al., 2012). Detailed analysis of DCM parameters revealed an early increase in connection strength from cSI to cSII cortex, and suggested that cSII might be the first cortical area to show a differential response dependent on stimulus detection. The backward cSII-cSI connection showed increased strength only for later data segments. In sum, this study corroborated the recurrent neural processing hypothesis of perceptual awareness (Lamme, 2006) for somatosensation and demonstrated that a dynamic interplay of early somatosensory areas can be effectively modeled in ERP data to study the neural mechanisms of tactile detection.

All of the above-mentioned studies used binary detection paradigms to study the neural underpinnings of tactile awareness. However, as detection paradigms do not separate awareness and cognitive stimulus processing, graded scales directly probing subjective reports of awareness measure conscious perception more 
A

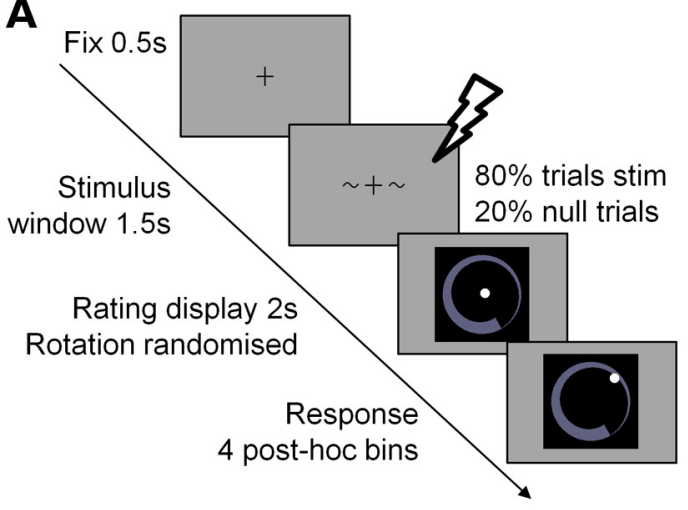

C

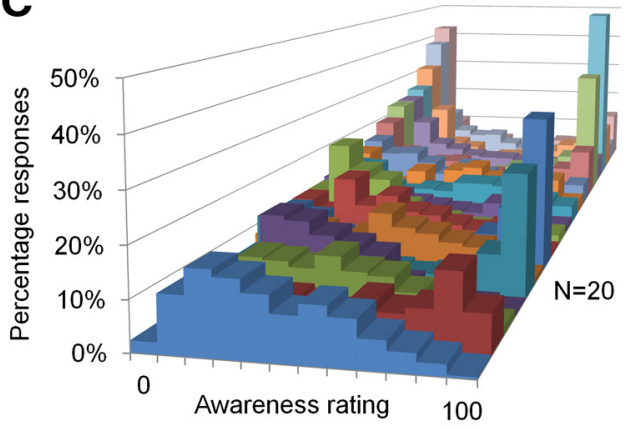

B
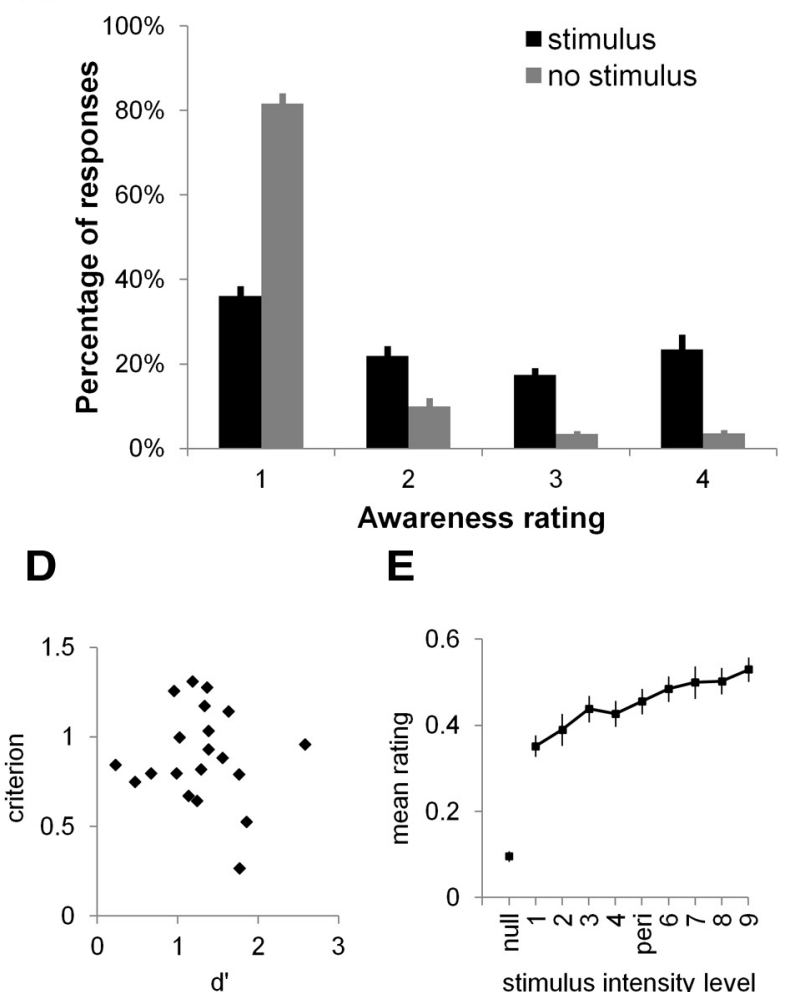

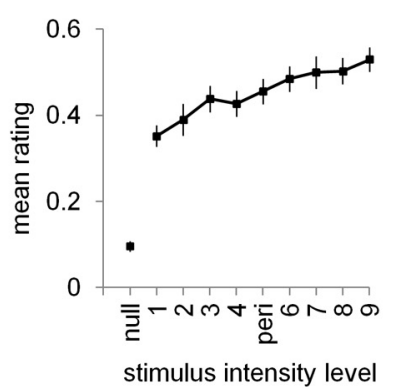

Figure 1. A, Experimental paradigm. Following perithreshold stimulation, participants indicated their responses on a circular gradient display. $\boldsymbol{B}$, Response histograms of trials with and without stimulation. Stimuli were assigned the lowest perceptual ratings in 37\% trials (82\% for null stimuli) and the highest in $23 \%$ trials (4\% null stimuli). Twenty-two stimuli (18\%) were given moderate ratings [(4\% null stimuli)]. Error bars represent SEMs. C, Individual response histograms of awareness ratings per participant. D, Individual proxies for $d^{\prime}$ and criterion, calculated after splitting trials into those with ratings below and above $50 \%$ respectively. $E$, Perceptual awareness ratings as a function of stimulus intensity (data from a separate psychophysical experiment). The leftmost data point represents mean ratings for the trials with no stimulation. Error bars represent SEMs.

exhaustively (Sandberg et al., 2010). In a neuroimaging study investigating the neural correlates of graded vision (Christensen et al., 2006), activity in the precentral gyrus, intraparietal sulcus, basal ganglia, and insula parametrically reflected perceptual clarity. Furthermore, a recent EEG study suggested that the P3 component reflects levels of visual awareness rather than cognitive components of stimulus processing (Salti et al., 2012). Until now, however, no study in the tactile modality has used a paradigm relying on explicit awareness reports rather than binary stimulus detection.

Here, we acquired EEG data in participants indicating their perceptual awareness of perithreshold tactile stimuli. We tested which ERP components parametrically reflect subjective stimulus awareness ratings, and which neuronal sources underlie the observed parametric effects despite identical physical stimulus intensities. Finally, we used DCM to infer whether recurrent processing between the identified areas is a sufficient parameter to explain a linear relation of neural activity and somatosensory awareness.

\section{Materials and Methods}

Participants. Twenty healthy participants (9 female; age range, 22-33 years; mean \pm SD age, $26.8 \pm 2.8$ years) enrolled in the study upon written informed consent. All subjects had normal or corrected-tonormal vision. The study was approved by the ethics committee of Charité-Universitätsmedizin Berlin and conformed to the Human Subjects Guidelines of the Declaration of Helsinki.

Stimuli and behavioral task. Electrical constant current pulse stimuli (duration, $0.2 \mathrm{~ms}$ ) were applied transcutaneously to the left median nerve (DS3; Digitimer) with a perithreshold intensity. Stimulation inten- sity was adjusted on practice trials (consisting of three short blocks of 20 trials) preceding the experiment to a level yielding a mean awareness rating of $\sim 50 \%$.

The experiment consisted of 800 trials divided into four blocks. Following a $500 \mathrm{~ms}$ fixation period, a visual cue was presented and remained on the display for $1500 \mathrm{~ms}$. In $80 \%$ of the trials, perithreshold stimuli (mean \pm SD intensity, $2.42 \pm 0.84 \mathrm{~mA}$ ) were applied 100-1400 ms after cue onset. In the remaining trials, no tactile stimulation was delivered. After each block, the stimulation intensity was readjusted to perithreshold intensity.

To rule out any fixed contingencies between the scaling of percepts and the response types, participants indicated responses on a polar display with its outer edge forming a circle (diameter, $\sim 8^{\circ}$ of the visual angle), and the inner edge an Archimedean spiral with polar coordinates $(r, \theta)$ described by the equation $r=0.98-0.05 \theta$ (Fig. $1 A$ ). The width of the display reflected the subjective rating of the percept quality and could increase in a clockwise or anticlockwise manner (randomized across trials). The thin end of the display corresponded to a complete lack of a tactile percept in a given trial, and the thick end to the presence of a clear tactile percept. Participants were asked to scale their responses on the whole continuum according to their perceptual awareness of the stimulus and were explicitly instructed not to assess the certainty of their assessment or the physical intensity of the stimulation. The display was rotated by 1 of $12^{\circ}\left(30-360^{\circ}\right.$ in steps of $\left.30^{\circ}\right)$, randomly determined in each trial. The responses were given after the visual stimulus disappeared by pressing the left mouse button with the right index finger. The maximum response interval duration was set to $2 \mathrm{~s}$, and no feedback on task accuracy was given. After the experiment, participants were asked how they scaled their responses and whether they used any segments to divide the rating continuum. Given that participants typically divided the rating continuum into four discrete segments, trials were binned into four rat- 
ing levels representing $0-25 \%, 25-50 \%, 50-75 \%$, and $75-100 \%$ perceptual awareness ratings, respectively.

Following the perceptual awareness task, an additional EEG localizer run was acquired. The tactile localizer consisted of 800 passively received suprathreshold stimuli (mean \pm SD intensity, $3.46 \pm 1.29 \mathrm{~mA}$ ) delivered with $2 \mathrm{~Hz}$ frequency and jittered with a random $0-10 \mathrm{~ms}$ delay. The intensity was set to a clearly suprathreshold level, but below motor threshold, and in case of participants' discomfort, to just below their subjective threshold of unpleasantness.

To substantiate that the participants indeed scaled their responses on the rating continuum according to their subjective perceptual state, we ran an additional psychophysical experiment with five male participants, two of whom had also participated in the main study. We administered two sessions of 300 trials in each session. The structure and timing of the trial was the same as in the main study. Here, however, we manipulated the physical intensity of the stimulus. In each session, we presented stimuli with nine different intensities (the perithreshold intensityestimated individually using a staircase procedure-as well as four subthreshold and four suprathreshold intensity). In one session, the stimulation intensities ranged from 96 to $104 \%$ of the perithreshold intensity (in milliamperes) in 1\% steps; in the other session, they ranged from 98 to $102 \%$ in $0.5 \%$ steps. The order of the sessions was randomized across participants. Furthermore, as in the main paradigm, 20\% of the trials did not contain any stimulation. In each trial, the participants were prompted to rate their subjective awareness of the stimulation and were instructed not to report the physical intensity of the stimulus or their response confidence. After completing the task, the participants were asked to assess how many intensity levels were administered in each session.

EEG recording and analysis. EEG signals were recorded using a 64channel active electrode system (ActiveTwo; BioSemi) with no reference (i.e., displayed against the common mode sense electrode). The locations of the electrodes, placed in an elastic cap conforming to a 10-10 system, were registered using an electrode-positioning system (CMS20S; Zebris Medical). EEG signals were sampled at $2048 \mathrm{~Hz}$ and downsampled offline to $512 \mathrm{~Hz}$. Continuous data were filtered off-line using high-pass $(>0.5 \mathrm{~Hz})$ and stopband $(48-52 \mathrm{~Hz})$ zero-phase forward and reverse Butterworth filters. Signals were re-referenced against an average reference. All analyses were performed using the SPM8 software package for EEG/MEG (Wellcome Trust Centre for Neuroimaging, Institute of Neurology, University College London, London, UK) and custom MATLAB code (The MathWorks).

ERP analysis. Data from the perceptual awareness task were epoched from $-100 \mathrm{~ms}$ before to $400 \mathrm{~ms}$ after stimulus onset and were baseline corrected to the prestimulus period. Eye movement artifacts were rejected by removing all trials containing amplitudes $>80 \mu \mathrm{V}$ and followed by a further careful visual inspection. Together, $15.37 \%$ of trials were rejected on average ( $\mathrm{SD}=11.63 \%)$.

Data were binned into four response levels, corresponding to awareness ratings of $0-25 \%, 25-50 \%, 50-75 \%$, and $75-100 \%$, respectively. Trials were averaged for the four rating levels separately using robust averaging, as implemented in SPM8. Mean signals were filtered using a $48 \mathrm{~Hz}$ low-pass filter. Data were converted into scalp-time images per participant and condition, and were smoothed over time with a Gaussian filter of $10 \mathrm{~ms}$ full-width at half-maximum.

Scalp-time maps were tested for significant differences across rating levels using an SPM-implemented second-level $4 \times 4$ flexible factorial design, with the two factors coding for block number and rating bin, and an additional covariate representing the physical intensity of the stimulation. Significance levels were inspected within the random field theory framework (Kilner et al., 2005) while correcting for multiple comparisons using the familywise error (FWE) correction procedure at the cluster level, thresholded at FWE $<0.005$ with a minimum cluster size of 15 scalp-time data points. To identify ERP components parametrically encoding perceptual awareness ratings, zero-centered linear contrasts were formed over the rating bin factor (contrast weights: $[-1.5,-0.5,0.5,1.5]$ for the positive and $[1.5,0.5,-0.5,-1.5]$ for the negative parametric contrast). All reported "positive" parametric effects could be attributed to ERP amplitude increases in the positive polarity for increasing rating levels, while "negative" parametric effects reflected amplitude increases in the negative polarity, as tested in a conjunction analysis of the parametric contrasts with polarity contrasts $(+1 /-1$ on all regressors; FWE, $<0.05$ ).

For the time windows showing significant effects in scalp-time space, a $3 \mathrm{D}$ source reconstruction procedure was applied per participant and rating level using a minimum-norm approach (Hämäläinen and Ilmoniemi, 1994; Litvak et al., 2011; independent identically distributed procedure under group constraints, as implemented in SPM8). The choice of the $3 \mathrm{D}$ source reconstruction approach was motivated by its relaxed symmetry priors compared with other methods. The time windows were identified based on the time latencies in scalp-time space for which peak significance levels were observed. The resulting 3D current density maps were smoothed with a $5 \times 5 \times 5$ full-width at half-maximum Gaussian kernel and entered into a one-way within-subjects ANOVA design with a rating bin factor (contrast weights: $[-1.5,-0.5,0.5,1.5]$ ). Significant effects were thresholded at FWE $<0.01$ (cluster level) with a minimum cluster size of 20 voxels.

Data from the localizer were epoched from $-50 \mathrm{~ms}$ before to $250 \mathrm{~ms}$ after stimulus onset and were baseline corrected to the prestimulus period. Eye movement artifacts were rejected following visual signal inspection. Together, $13.65 \%$ of trials were rejected on average $(\mathrm{SD}=5.99 \%)$. Trials were averaged using robust averaging.

Dynamic causal modeling. DCM was used to infer hidden parameters of neuronal models explaining the observed EEG data (David et al., 2006). DCM models the data using a network of a few dynamically interacting sources, here implemented as equivalent current dipoles. The sources can be (1) connected in a context-independent way, modeling fixed effective connectivity between regions; (2) connected in a contextdependent manner, representing modulatory influences; or (3) driven by external input.

Nine structural models were specified for the same architecture with five sources: a contralateral (i.e., right) SI dipole (cSI), a symmetrical SII dipole pair (cSII and iSII), and a symmetrical dipole pair in the premotor cortex (cPMC and iPMC; Romo et al., 1993; Thees et al., 2003). Equivalent current dipoles were fitted to localizer data (cSI, c/iSII) and task data (cPMC/iPMC) along the variational Bayesian scheme (Kiebel et al., 2008), taking negative free energy as the approximation to model evidence (log evidence), thus determining the probability of observing the data given a model (Stephan et al., 2009). Per participant, the dipoles were fitted 10 times iteratively, at each step selecting the dipole with maximum negative free energy, using the following procedure: (1) a single dipole in CSI was fitted to the entire poststimulus epoch of the localizer data, bound by prior location coordinates from individual 3D source reconstruction of the N20 component (prior location variance $10 \times 10 \times 10 \mathrm{~mm}$ ); (2) a symmetrical SII dipole pair was added to the optimized SI dipole and fitted to the poststimulus localizer data, with location priors based on individual 3D source localization of the $80-125$ ms segment of the localizer (Hagiwara et al., 2010) and $10 \times 10 \times 10 \mathrm{~mm}$ prior location variance; and (3) a symmetrical PMC dipole pair was added to the three dipoles determined in the steps above and fitted to the entire poststimulus epoch of the task data, with location priors set to the peak voxel of the PMC cluster identified in the $3 \mathrm{D}$ source reconstruction of the $\mathrm{P} 300$ component $(10 \times 10 \times 10 \mathrm{~mm}$ prior location variance; Fig. $2 B$ ).

The structural connectivity of the models is shown in Figure 3A. Only cSI was assumed to be directly driven by external input, with its onset set to $20 \mathrm{~ms}$, simulating the N20 component. Each model allowed for a different subset of connections to be parametrically modulated by perceptual awareness level, i.e., to change the coupling strength between regions proportionally to the perceptual awareness rating (connection weights: $[-1.5,-0.5,0.5,1.5])$. The models were divided into three families, assuming different connectivity patterns. In the local connectivity models, cSI and c/iSII were interconnected with modulatory connections, while cPMC/iPMC were disconnected from the other areas, which precluded their influence on the behavior of the models but allowed for a direct comparison with other models. The proximal connectivity models allowed for all five sources to be interconnected, but only assumed modulation between cSI and cSII/iSII. The global connectivity 

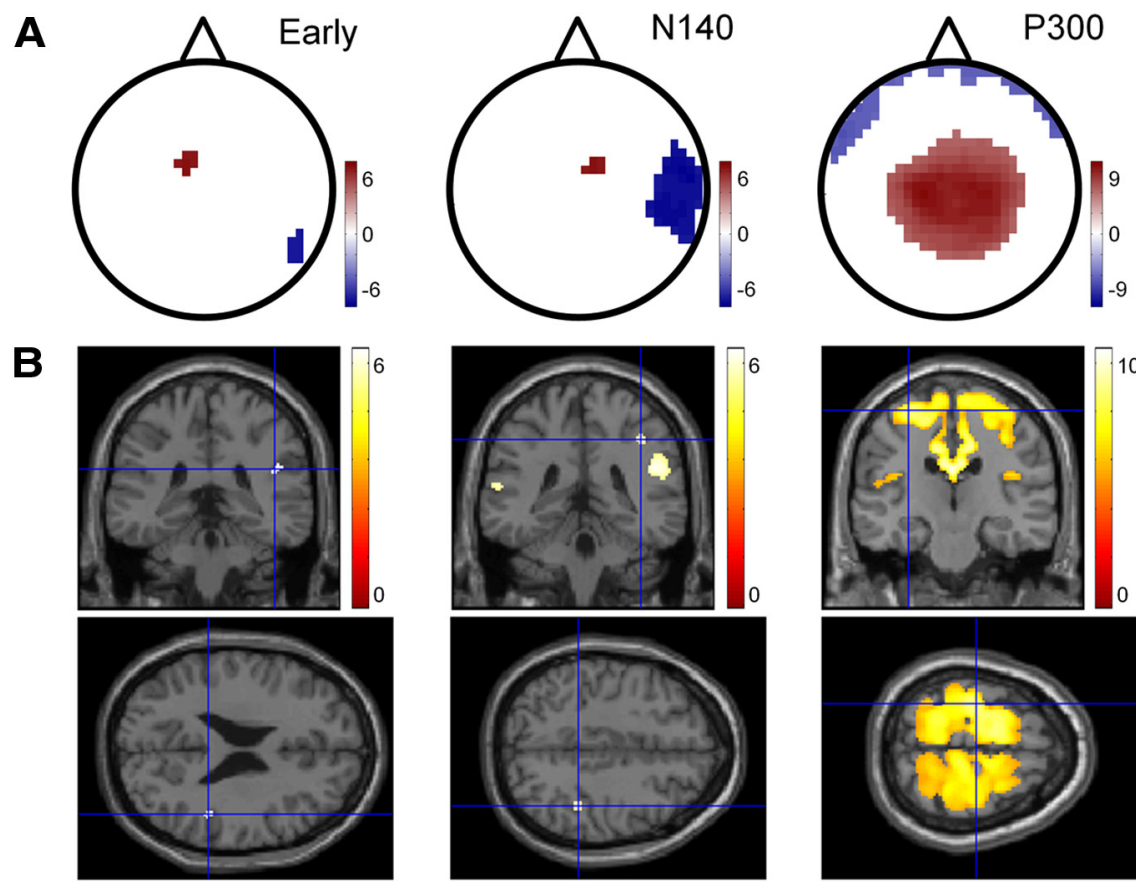

C
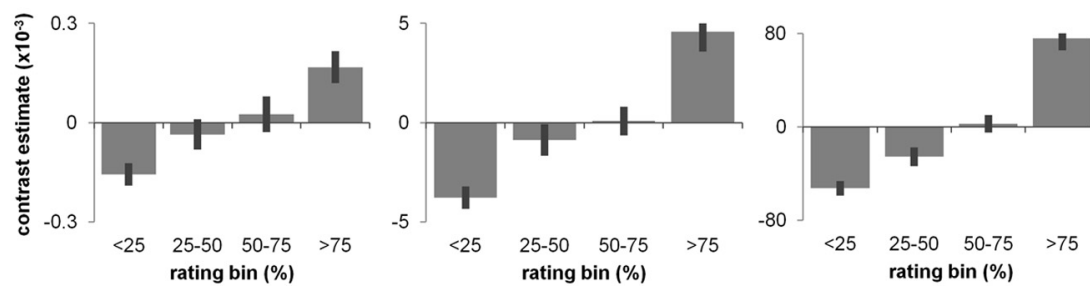

Figure 2. Significant parametric effects of perceptual awareness rating. $A$, Awareness-related parametric effects on ERP topographies. Contrasts parametrically coding perceptual rating were significant $(F W E,<0.005)$ for three time windows only, representing early (86-92 ms) ERP divergence, N140 (131-186 ms), and P300 (211-391 ms). Averaged thresholded $t$ values shown for three time windows; color marks ERP polarity. $B, 3 D$ source reconstructions for the windows of interest showing parametric effects in ERPs. $t$ values of the parametric $t$ test are shown across perceptual awareness rating levels (FWE, $<0.01$ ). Left, Early activation (86 ms), slice centered on CSII, MNI (46, -36, 24). Middle, N140 component (131-158 ms), slice centered on SI, MNI (40, -38, 44). Right, P300 component (211-391 ms), slice centered on PMC, MNI (-30, -20,54). C, Contrast estimates for the respective coordinates and latencies, as indicated above. Error bars mark $90 \%$ confidence intervals.

models were interconnected with modulatory connections among all areas.

The models were compared using a random-effects Bayesian model selection (BMS) procedure (Stephan et al., 2010), which is robust to outliers (Stephan et al., 2009) and allows choice of the best model based on its log evidence, estimating the probability of observing single-subject data, given the model. Here, given a large number of models, our analysis consisted of two steps. In the first step, we used a family inference procedure (Penny et al., 2010) to choose among three families of models, containing the local, proximal, and global connectivity patterns, respectively (Fig. 3B). In the second step, we applied BMS to models within the winning family to infer the exact type of connections (i.e., feedforward, backward, or recurrent) parametrically modulated by perceptual awareness (Fig. 3C,D). Both steps were performed for four time epochs, starting at $1 \mathrm{~ms}$ and with lengths ranging from 100 to $400 \mathrm{~ms}$ in steps of $100 \mathrm{~ms}$ (Garrido et al., 2007; Auksztulewicz et al., 2012).

Time-frequency analysis. Both power and phase information were analyzed in the time-frequency domain, aiming at a replication of previous findings indicating the effects of prestimulus power and phase on subsequent visual detection (Busch et al., 2009; Mathewson et al., 2009). Continuous data were re-epoched from $-500 \mathrm{~ms}$ before until $500 \mathrm{~ms}$ after stimulus onset and were decomposed into time-frequency data using a sliding window fast Fourier transform (single Hanning taper with an adaptive window length of three cycles in steps of $10 \mathrm{~ms}$; frequency range, $8-48 \mathrm{~Hz}$ in steps of $2 \mathrm{~Hz}$ ). To ensure that the baseline period is not contaminated by visual evoked responses due to the visual cues presented before and after the stimulation window, we restricted our timefrequency analysis to trials in which stimuli were presented with latencies $>600 \mathrm{~ms}$ and $<1000 \mathrm{~ms}$. For each time-frequency point, power and phase information were calculated per trial and participant. Two series of repeated-measures ANOVAs were performed on power estimates: one for post-stimulusinduced power and one for prestimulus power. In the poststimulus power analysis, the amplitude values $A$ were rescaled for each trial $k$, time point $t$, and frequency $f$ to the prestimulus period (i.e., for time points preceding stimulus onset $t_{0}$ ) using logarithmic ratio rescaling,

$$
\begin{aligned}
A_{k(t, f)}^{\prime}=10 \cdot\left(\log _{10} A_{k(t, f)}\right. & \\
& \left.\quad-\frac{1}{t_{0}} \sum_{t=1}^{t_{0}} \log _{10} A_{k(t, f)}\right),
\end{aligned}
$$

and were averaged over trials for each awareness rating bin separately. In the prestimulus power analysis, time-frequency data were averaged without rescaling. Both prestimulus and poststimulus power estimates were analyzed separately for each frequency band: alpha $(8-12 \mathrm{~Hz})$, lower beta $(12-20 \mathrm{~Hz})$, upper beta $(20-30 \mathrm{~Hz})$, and gamma $(30-48 \mathrm{~Hz})$.

The prestimulus phase information was investigated in two analyses. In the first analysis, to replicate the previous reports of prestimulus phase effects on subsequent stimulus detection (Mathewson et al., 2009), trials were binned into four cells by their average prestimulus power (median split of log-transformed power averaged across time points from $-200 \mathrm{~ms}$ until stimulus onset) and instantaneous phase angles at stimulus onset (ranging between $45^{\circ}$ and $225^{\circ}$ vs between $225^{\circ}$ and $45^{\circ}$ ). Mean awareness ratings were computed for the opposite phase angles and prestimulus power estimates per participant, frequency, and EEG channel. The effects of prestimulus phase, as well as its interaction with mean prestimulus power, on subsequent awareness rating were analyzed using a series of two-way repeated-measures ANOVAs performed per EEG channel and frequency, with significance controlled for multiple comparisons using a false discovery ratio (FDR) of 0.05 (Benjamini and Hochberg, 1995).

In the second analysis, the instantaneous phase angles, obtained for each trial and participant, were used to calculate intertrial coherence values per channel, frequency, time point, trial, and participant (Busch et al., 2009). To specifically investigate the possible effects of prestimulus phase $\varphi$ on awareness ratings, we quantified the phase distribution differences between the lowest and highest rating bins by computing phase bifurcation index (PBI) values (Busch et al., 2009) for each channel, $f$, prestimulus $t$, and participant:

$$
\operatorname{ITC}_{(t, f)}=\frac{1}{n} \sum_{n=1}^{k} e^{i\left(\varphi_{k(t, f)} 2 \pi\right)}
$$

$\operatorname{PBI}_{(t, f)}=\left(\operatorname{ITC}_{\text {lowest }(t, f)}-\operatorname{ITC}_{\text {all }(t, f)}\right) \times\left(\operatorname{ITC}_{\text {highest }(t, f)}-\operatorname{ITC}_{\text {all }(t, f)}\right)$.

The PBI is close to 0 under the null hypothesis, takes positive values when different conditions exhibit phase locking at opposite angles, and takes negative values when only one condition is phase locked. To test whether PBI was present in the data, a series of tests based on permutation statistics was performed per channel, frequency, and time point, while con- 


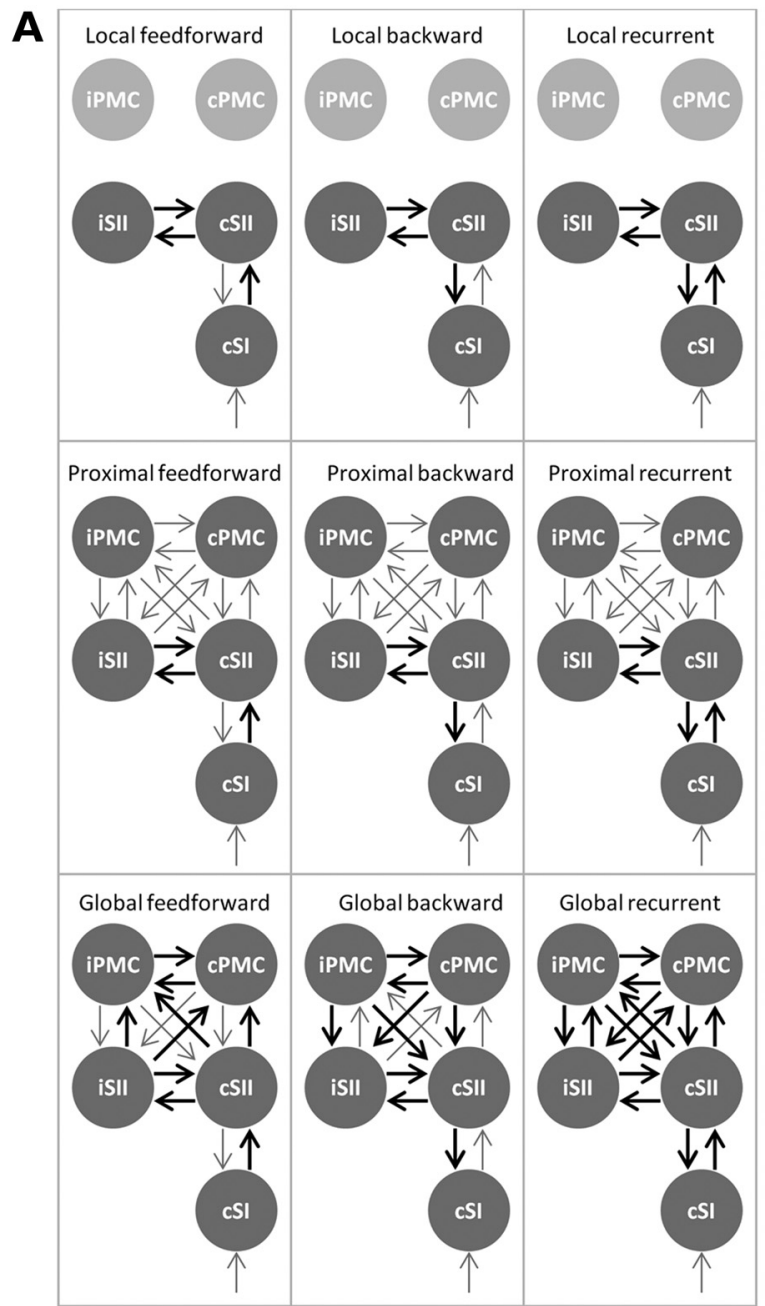

B

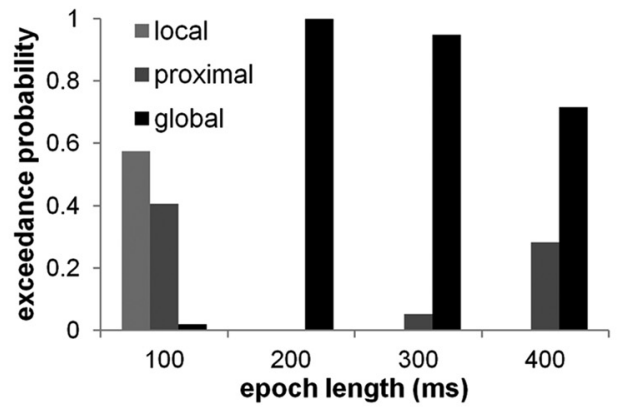

C 1 aforward

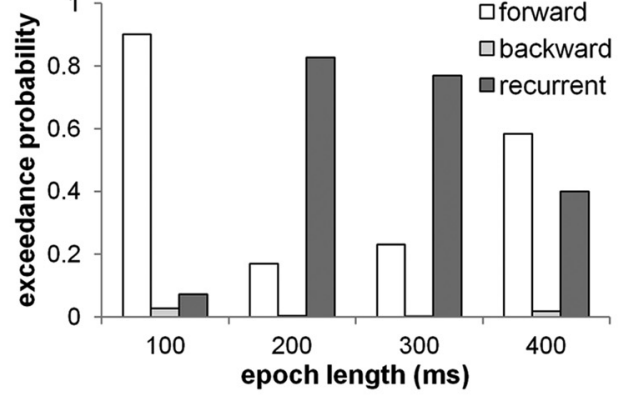

D

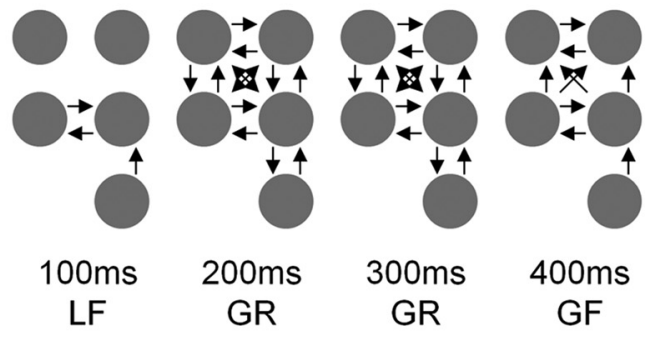

Figure 3. Dynamic causal modeling. $\boldsymbol{A}$, Nine models were constructed for the same architecture containing five anatomical sources modeled as dipoles (CSI; c/iSll; c/iPMC). Sensory input was assumed to directly reach only $\mathrm{CSI}$. Thin gray arrows mark fixed connections, whose strength was not modulated by conditions. Bold black arrows mark modulatory connections, whose strength changed parametrically with increasing perceptual awareness rating. B, Familywise Bayesian model selection (random effects) among local, proximal, and global connection patterns for four time epochs. C, Random-effects Bayesian model selection for four time epochs within the respective winning model families. $\boldsymbol{D}$, Summary of the winning models for four time epochs. LF, Local feedforward; GR, global recurrent; GF, global feedforward.

trolling for multiple comparisons using FDR $=0.05$ (Benjamini and Hochberg, 1995). Specifically, pseudo-PBI values were calculated from reshuffled data in each participant and used to calculate a grand mean across participants. The grand mean based on reshuffled data was then compared against the observed grand mean. This procedure was repeated 1000 times, resulting in exact $p$ values defined as the proportion of grand means based on reshuffled data that exceeded the observed values.

\section{Results}

\section{Behavioral results}

Perceptual awareness rating distributions clearly differed between trials with and without stimulation $\left(t_{(1,19)}=11.9676, p<\right.$ 0.001 ; Fig. $1 B$ ). Most participants (17 of 20 ) divided the rating continuum into discrete segments, i.e., drew across the circular gradient or used a number of response categories descriptively, with the median number of segments equal to 4 . Thus, trials were binned into four rating levels (see Materials and Methods). Due to the rating distribution (Fig. 1C), the number of trials in each rating bin could potentially have been unbalanced; in a control analysis, data were subsampled for a matching number of trials across rating bins (see below).

\section{Event-related potentials}

The earliest ERP activity parametrically encoding perceptual awareness level was observed at 86 ms poststimulus over contralateral parietal channels for the negative parametric contrast (i.e., parametric amplitude increases in the negative polarity; see Materials and Methods) and at $92 \mathrm{~ms}$ ipsilateral central channels for the positive parametric contrast. These early parametric effects were followed by a significant parametric effect in the N140 component. Finally, ERPs parametrically encoded perceptual awareness ratings in the P300 component. No significant effects were found for block number or intensity ( $F$ tests; FWE, 0.1). Table 1 summarizes all significant clusters for which parametric effects of awareness rating were observed.

To balance the number of trials in each condition in a control analysis (Fig. 1C), the binned ERP data were reanalyzed using subsampling. The results of this analysis were strikingly similar to the previous analysis using all data, and only minor differences in the peak latencies of the three ERP components parametrically reflecting awareness ratings were observed. 
Table 1. Parametric effects of awareness ratings on ERP amplitude

\begin{tabular}{|c|c|c|c|c|c|c|c|c|c|}
\hline \multirow[b]{2}{*}{ Contrast } & \multirow[b]{2}{*}{ Component } & \multicolumn{3}{|c|}{ Cluster level } & \multicolumn{2}{|c|}{ Peak level } & \multicolumn{3}{|c|}{ Peak coordinates } \\
\hline & & $p_{\text {FWE }}$ & $N$ voxels & Latency range (ms) & $p_{\text {FWE }}$ & $t$ & $x(\mathrm{~mm})$ & $y(\mathrm{~mm})$ & Time (ms) \\
\hline \multirow[t]{3}{*}{ Positive parametric } & Early & $<0.001$ & 19 & 86 & 0.001 & 5.68 & -9 & 8 & 92 \\
\hline & N140 & $<0.001$ & 19 & 186 & 0.002 & 5.51 & 9 & 2 & 186 \\
\hline & P300 & $<0.001$ & 10,082 & $211-391$ & 0 & 11.85 & 13 & -14 & 330 \\
\hline \multirow[t]{3}{*}{ Negative parametric } & Early & $<0.001$ & 18 & 92 & 0.002 & 5.36 & 47 & -46 & 86 \\
\hline & N140 & $<0.001$ & 481 & $131-158$ & 0 & 6.47 & 55 & 2 & 131 \\
\hline & P300 & $<0.001$ & 1245 & $301-381$ & 0 & 6.66 & -55 & 18 & 324 \\
\hline
\end{tabular}

Significant clusters and their respective maxima in scalp-time space ( $p_{\mathrm{FWE}}<0.005$; cluster level, minimum cluster size, 15 voxels; contrast weights: $\left.[-1.5,-0.5,0.5,1.5]\right)$. Positive parametric effects can be attributed to ERP amplitude increases in the positive polarity for increasing rating levels, while negative parametric effects reflect amplitude increases in the negative polarity.

\section{Control psychophysical experiment}

To investigate whether participants scaled their responses along their subjective perceptual continuum, we ran an additional psychophysical experiment where we manipulated the physical intensity of tactile stimulation. The participants' responses were averaged for each participant, session, and intensity level, and analyzed in a repeated-measures ANOVA with two factors, coding for stimulation intensity (nine levels corresponding to the different stimulation intensities) and session (high vs low range of stimulation intensities), respectively. The analysis revealed a significant effect of stimulation intensity $\left(F_{(9,85)}=26.4218, p<\right.$ $0.001)$ but no significant effect of session $(p=0.39)$. An additional analysis of covariance revealed that participants' ratings covaried with the stimulation intensity (treated as an ordinal covariate, collapsed across sessions; $F_{(1,80)}=45.3229, p<0.001$; slope estimate, $0.0205, p<0.001$; Fig. $1 E$ ). Asking the participants post hoc about the number of perceived intensity levels revealed that all participants distinguished fewer intensity levels than actually presented (median 5).

\section{Control analyses}

Several control analyses were performed to test alternative interpretations of the observed parametric effects on ERPs. First, as the results could arguably be influenced by changes in criterion rather than only arise due to modulation in perceptual awareness, the possible effects of criterion on ERPs were investigated. Given that the signal detection theory categories (hits, misses, false alarms, correct rejections) could not be applied to continuous responses, the trials were split into those with ratings above and below $50 \%$, respectively. This was done separately for trials with and without stimulation. The signal detection theory formulas were applied per participant to compute proxies for $d^{\prime}$ and criterion in each of the 200-trial blocks (Fig. 1D). The $d^{\prime}$ and criterion proxy values were then entered into a repeated-measures multivariate ANOVA, indicating that while no systematic shifts in criterion occurred between the blocks (Wilks' lambda $=0.562$, $\left.F_{(6,14)}=1.817, p=0.168\right)$, the between-subjects multivariate effects were significant (Wilks' lambda $=0.047, F_{(2,18)}=180.487$, $p=0)$. Univariate tests revealed that participants differed with respect to both their criteria $\left(F_{(19,57)}=2.6805, p=0.002\right)$ and $d^{\prime}$ values $\left(F_{(19,57)}=2.0477, p=0.019\right)$. Thus, as we wanted to test the possible effects of criterion on the observed ERP effects, in the next step the participants were split into those adopting high criterion (above median, criterion proxies averaged across blocks) versus low criterion (below median), and a parametric analysis of ERP topographies was run with a binary betweensubjects variable coding for criterion differences. This analysis revealed that the parametric effects on ERPs were preserved and occurred at the same latencies and topographies as in the original analysis with all participants, and no differences were observed between the high-criterion and low-criterion participants (FWE,
$>0.05)$. An identical pattern of results was observed for the analysis comparing participants with above-median and belowmedian $d^{\prime}$ values. Therefore, the differences between participants in their adopted criterion and their sensitivity to the perithreshold stimulus are unlikely candidates for explaining the reported results on ERPs.

In a further control analysis, to test whether the later observed effects (in N140 and P300) might be merely a consequence of the earlier effects or whether they each explain unique variance, two masks were created containing time-space "voxels" for which the significant parametric effects were observed in the early time window ( $\sim 90 \mathrm{~ms}$ ) and N140 component, respectively. The two masks were used to extract for each participant and condition (i.e., awareness rating) the average ERP amplitude from the SPMconverted topography images. The extracted average ERP amplitudes served as covariates in a repeated analysis of the parametric effects on ERPs. First, we introduced only one covariate coding the amplitude at $\sim 90 \mathrm{~ms}$, and tested whether the parametric effects on N140 and P300 can still be observed. Second, both covariates (coding $\sim 90 \mathrm{~ms}$ and N140, respectively) were entered, allowing the testing of whether the parametric effects on P300 can be observed. This analysis revealed that the parametric effects on P300 are robust and explain variance independent from the amplitude of both the early components at $\sim 90 \mathrm{~ms}$ and the N140 component. The N140 component itself still parametrically reflected awareness ratings when controlling for ERP amplitude at $\sim 90 \mathrm{~ms}$. Thus, the parametric effects in the three time windows were largely independent of one another.

Finally, as the stimuli were presented with randomized latencies in a $1500 \mathrm{~ms}$ time window, the effects of temporal expectation of the upcoming stimulus on the evoked ERPs were assessed by performing a median split of the trials into those with short and long stimulus latencies, respectively, in addition to binning the trials by their corresponding awareness rating. Behaviorally, stimuli presented later in time were assigned higher awareness ratings, as revealed in a paired $t$ test $t_{(2,19)}=-2.7135, p=$ 0.0138 ). The analysis of the effects of stimulus latency on ERPs revealed differences in the late components $(209-400 \mathrm{~ms}$; $F$ test, FWE, $<0.005$, minimum cluster size 15 scalp-time voxels) between stimuli presented at short and long latencies. The late components of ERPs evoked by stimuli presented early into the time window showed a predominantly posterior topography, while those evoked by stimuli with longer onsets showed a more anterior topography (separate $t$ tests, FWE, $<0.005$; minimum cluster size 15 scalp-time voxels). This topography shift could be localized to a source in the left intraparietal sulcus (peak voxel MNI coordinates $[-46,-42,22]$, as labeled using SPM Anatomy Toolbox; Eickhoff et al., 2005) with a stronger activity estimate for stimuli presented at longer latencies $\left(p_{\mathrm{FWE}}=0.008\right.$ cluster level; $t=4.78$; cluster size, 37 voxels). Independent of the effects of stimulus onset, the parametric awareness rating effects on 
Table 2. Parametric effects of awareness ratings on estimated activity of reconstructed sources

\begin{tabular}{|c|c|c|c|c|c|c|c|c|c|}
\hline \multirow[b]{2}{*}{ Time window (ms) } & \multirow[b]{2}{*}{ Area } & \multirow[b]{2}{*}{ Probabilistic label (cluster) } & \multicolumn{2}{|c|}{ Cluster level } & \multicolumn{2}{|c|}{ Peak level } & \multicolumn{3}{|c|}{ Peak coordinates } \\
\hline & & & $p_{\mathrm{FWE}}$ & $N$ voxels & $p_{\mathrm{FWE}}$ & $\mathrm{T}$ & $x(\mathrm{~mm})$ & $y(\mathrm{~mm})$ & $z(\mathrm{~mm})$ \\
\hline 86 & cSII & $\mathrm{OP} 1, \mathrm{R}$ & 0.007 & 29 & 0.005 & 5.21 & 46 & -36 & 24 \\
\hline \multirow[t]{3}{*}{92} & cSII & $\mathrm{OP} 1, \mathrm{R}$ & 0.001 & 250 & 0.001 & 5.88 & 46 & -36 & 22 \\
\hline & & OP4/OP1, R & 0.002 & 220 & 0.003 & 5.45 & 56 & -14 & 18 \\
\hline & & $\mathrm{OP} 3, \mathrm{R}$ & 0.005 & 50 & 0.003 & 5.43 & 38 & -6 & 14 \\
\hline \multirow[t]{6}{*}{ 131-158 } & iSII & OP4/OP1, L & 0.002 & 142 & 0 & 5.37 & -62 & -16 & 20 \\
\hline & cSII & IPC/OP1, R & 0.001 & 227 & 0.001 & 5.2 & 50 & -38 & 26 \\
\hline & CSII/CSI & OP3/BA3a, R & 0.004 & 63 & 0.001 & 5.12 & 52 & -16 & 26 \\
\hline & cSl & Area $2, R$ & 0.006 & 24 & 0.001 & 5.11 & 40 & -38 & 44 \\
\hline & iSII & $\mathrm{OP1}, \mathrm{L}$ & 0.005 & 30 & 0.002 & 4.97 & -56 & -26 & 16 \\
\hline & iSII? & TE3, L & 0.002 & 105 & 0.003 & 4.96 & -60 & -38 & 12 \\
\hline 186 & iSII & $\mathrm{OP} 4, \mathrm{~L}$ & 0.004 & 41 & 0.004 & 5.47 & -58 & -18 & 14 \\
\hline \multirow[t]{4}{*}{ 211-391 } & c/iPMC & $\mathrm{BA6}, \mathrm{R} / \mathrm{L}$ & 0 & 18,152 & 0 & 10.56 & 0 & -28 & 24 \\
\hline & iSII & IPC/OP1, L & 0 & 508 & 0 & 8.77 & -40 & -34 & 18 \\
\hline & cSII & IPC/OP1, R & 0 & 406 & 0 & 7.41 & 38 & -30 & 18 \\
\hline & iSII? & V5/IPC, L & 0 & 561 & 0 & 6.46 & -40 & -74 & 14 \\
\hline
\end{tabular}

Significant clusters, their anatomical labels based on cytoarchitectonic probabilistic maps, and respective peak voxels $\left(\mathrm{p}_{\mathrm{FWE}}<0.01\right.$ cluster level; minimum cluster size, 20 voxels; contrast weights: $\left.[-1.5,-0.5,0.5,1.5]\right) .0 \mathrm{P}$, Parietal operculum; IPC, inferior parietal cortex; TE3, temporal auditory cortex, area 3; R, right; L, left.

ERPs were preserved in this analysis and closely resembled the results of the original analysis in terms of topography, latencies, and underlying sources.

\section{D source reconstructions}

Sources underlying the above parametric ERP effects were investigated for four time windows, defined by the latencies at which significant parametric effects were observed in the ERP analysis (Fig. 2 B, C; Table 2). For the early time windows (86 and $92 \mathrm{~ms}$ ), only sources in CSII were identified. Later, in the first of the midlatency time windows (131-158 ms), the parametric effect was assigned to c/iSII and cSI. In the $186 \mathrm{~ms}$ time window, only a source in iSII was active. Finally, in the late time window (211$391 \mathrm{~ms}$ ), besides c/iSII and cSI, a broad network of sources was evident, including bilateral Brodmann area 6 (i.e., encompassing both the supplementary motor areas and the premotor cortices). All reported brain areas were identified using the cytoarchitectonic probabilistic maps in SPM Anatomy Toolbox (Eickhoff et al., 2005) by overlapping anatomy and statistic parametric maps of the awareness rating contrasts and selecting the most probable label for each activity cluster. Please note that for the sake of comprehensibility and given the striking similarity between the adjacent time windows identified for opposing polarities, the source reconstructions of the two "early" time windows ( 86 and $92 \mathrm{~ms}$ ) as well as the two N140 time windows (131-158 and $186 \mathrm{~ms}$ ) are summarized on single panels of Figure 2, $A$ and $B$, respectively.

\section{Dynamic causal modeling}

The Bayesian model selection procedure among the nine alternative models (Fig. $3 A$ ) contained two steps. In the first step (Fig. $3 B$ ), random-effects familywise BMS (group level, $N=20$; Stephan et al., 2010) was used to identify the most likely connectivity pattern between the five sources. Three model families - with local, proximal, and global connectivity patterns, respectively-were compared for four time epochs, all starting at $1 \mathrm{~ms}$ and with lengths ranging from 100 to $400 \mathrm{~ms}$ in steps of $100 \mathrm{~ms}$. For the first time window (100 ms), the local connectivity family showed the highest exceedance probability (i.e., the probability that the model is more likely than other models, given the group data), followed by the proximal and global connectivity families, respectively. For the three later time windows (200-400 ms), the global connectivity family outperformed the others. In particu- lar, the global connectivity family gained strong evidence for the 200 and $300 \mathrm{~ms}$ data segments, with exceedance probability $>95 \%$.

In the second step (Fig. 3C), the winning model families from each time window were chosen for within-family random-effects BMS (group level, $N=20$; Stephan et al., 2010). Here, choosing among local connectivity models for the $100 \mathrm{~ms}$ time window, the model allowing for feedforward modulation clearly outperformed the others ( $>90 \%$ exceedance probability). For later data segments, choosing from global connectivity models, the global recurrent model was the winning model for the 200 and $300 \mathrm{~ms}$ time windows, and the global feedforward model had the highest exceedance probability for the $400 \mathrm{~ms}$ segment (Fig. 3D). Note, however, that for the longest data segment the winning feedforward model was closely followed by a global recurrent model (exceedance probabilities, 58 and $41 \%$, respectively).

\section{Time-frequency analysis}

The analysis of time-frequency power estimates, performed per frequency band and for both prestimulus and poststimulus power revealed no significant parametric effects corresponding to subsequent awareness rating (SPM8; random field theory framework; Kilner et al., 2005; corrected for multiple comparisons at FWE $=0.05)$. In the analysis of prestimulus phase as well as its interaction with prestimulus power (Mathewson et al., 2009), a series of two-way repeated-measures ANOVAs per frequency and channel revealed that none of these tests reached significance when correcting for multiple comparisons using FDR $=0.05$ (Benjamini and Hochberg, 1995). A series of permutation tests on phase bifurcation index values (Busch et al., 2009) performed per channel, frequency, and time point, with significance controlled for multiple comparisons using FDR $=0.05$, did not indicate any significant differences in phase locking between trials with highest and lowest awareness ratings, respectively.

\section{Discussion}

To investigate whether the ERP dynamics associated with conscious somatosensation parametrically reflect the different levels of perceptual awareness, we analyzed EEG data acquired from participants rating their subjective awareness of perithreshold tactile stimuli. Our results are in line with previous findings indicating that conscious detection of weak tactile stimuli is characterized by an early divergence of ERP responses between 
detected and missed stimuli (Schubert et al., 2006; Jones et al., 2007; Auksztulewicz et al., 2012). However, this study is the first to show that ERP components reflect graded tactile awareness parametrically.

Early SII activity parametrically reflects perceptual awareness The short latency ( $\sim 90 \mathrm{~ms}$ ) of the first ERP components parametrically encoding tactile awareness corresponds to the latency of the ERP divergence in tactile detection paradigms (Schubert et al., 2006; Jones et al., 2007; Auksztulewicz et al., 2012). Our source localization results clearly point to the secondary somatosensory cortex as the origin of this early activity, consistent with single-neuron recordings in macaques (de Lafuente and Romo, 2006), where neurons in SII and areas downstream, but not in SI, allowed for an above-chance prediction of the monkeys' decisions in a tactile detection paradigm. Furthermore, DCM results for the early data segment ( $<100 \mathrm{~ms}$ ) show that parametric modulation of the forward cSI-cSII connection strength is sufficient to explain the observed ERP effects.

Among the possible explanations for the parametric relation between early SII activity and subsequent perceptual awareness ratings, top-down attentional modulation could be invoked. Early awareness-related responses have been shown to be modulated by attention (Koivisto and Revonsuo, 2008), and more generally attention was suggested to influence early poststimulus ERP components (for review, see Luck et al., 2000). It has also been demonstrated that synchronized neuronal firing in SII is sensitive to attentional modulation (Steinmetz et al., 2000), which in our data could result in a parametric modulation of ERP amplitude attributed to SII activity. However, we found no evidence for any frontoparietal activity during or preceding this early time window, as would have been expected by an attentional effect (Corbetta and Shulman, 2002; Bressler et al., 2008; Greenberg et al., 2010; Petersen and Posner, 2012).

It could be argued that top-down attentional modulation might not share the parametric relation between ERP amplitude and subsequent awareness rating, but would rather be detectable as changes in oscillatory power. Several studies reported that prestimulus baseline oscillations have electrophysiological and behavioral relevance for poststimulus tactile processing in both attentional manipulation paradigms (Anderson and Ding, 2011; Haegens et al., 2011) and measurements of spontaneous prestimulus activity in detection paradigms (Linkenkaer-Hansen et al., 2004; Busch et al., 2009; Mathewson et al., 2009; Schubert et al., 2009; Zhang and Ding, 2010). However, an analysis of prestimulus and peristimulus time-frequency predictors of subsequent perceptual report yielded no significant results.

Alternatively, the parametric effects observed at this early latency might be a marker of signal amplification associated with conscious stimulus processing and independent of attention (Lamme, 2006; Wyart and Tallon-Baudry, 2008; Tallon-Baudry, 2011). Given that the early effects could be localized to SII and not SI, such signal amplification might possibly be implemented in SI by a superposition of neural activity corresponding to stimulus processing with a stochastic variation of neuronal firing, resulting in a varied length of continuous firing, which then in turn might be integrated in SII and result in the observed parametric effects on the amplitude of SII activity (de Lafuente and Romo, 2006). Thus, SII might play a role in conscious touch similar to that in high-order areas of vision (i.e., the fusiform gyrus, lateral occipital area, and intraparietal sulcus), whose BOLD activity has been shown to reflect subjective visibility ratings but not objective per- formance (Hesselmann and Malach, 2011; Hesselmann et al., 2011).

\section{Awareness-related activity in sensorimotor network can be explained by recurrent processing}

The parametric awareness-related effects observed at latencies $<200 \mathrm{~ms}$ could be localized to the somatosensory cortex, namely the bilateral SII and contralateral SI. A recent study on EEG correlates of drug-induced loss of consciousness found that early stimulus-related time-frequency responses found in the 16-20 $\mathrm{Hz}$ range, 30-110 ms poststimulus, and localized to cSI were already diminished by progressive sedation (Supp et al., 2011), although another early cSI response in a higher frequency range was preserved across sedation levels. Similarly, several studies in the visual domain found that only the activity in sensory regions in the occipital cortex distinguished between early responses to physically identical seen and missed stimuli (Pins and Ffytche, 2003; Liu et al., 2012). The finding of parametric consciousnessrelated effects occurring in the somatosensory areas at early latencies is in line with theoretical accounts stressing the role of localized sensory activity in phenomenal awareness (Zeki, 2001; Lamme, 2006; Block, 2007).

Previously, we have reported that recurrent neural processing between SI and SII can explain the observed ERP differences between consciously and unconsciously processed stimuli in tactile detection (Auksztulewicz et al., 2012). That finding allowed for an extrapolation of the recurrent processing hypothesis of perceptual awareness (Lamme, 2006), largely based on studies in vision (Pascual-Leone and Walsh, 2001; Ro et al., 2003; Boehler et al., 2008; Fahrenfort et al., 2008), to the somatosensory modality. The dynamic causal modeling results presented here replicate our previous study and offer a further extension of the role of recurrent processing onto graded perceptual awareness ratings. The models allowing for a parametric change in connection strengths of the feedforward-feedback loops within the somatosensory cortex (cSI-c/iSII), and between the somatosensory areas and PMC, were chosen when longer data segments (200-300 ms) after the initial feedforward sweep $(<100 \mathrm{~ms})$ were analyzed. This is supported by the results of a 3D source reconstruction of the N140 components, showing a significant parametric activation of cSI related to subsequent perceptual awareness ratings and possibly reflecting awareness-related reentrant processing in cSI. Together, our findings speak to the notion that the strength of recurrent processing within the system might be linearly related to the quality of the percept.

Also the network of areas identified as parametrically reflecting somatosensory awareness ratings in the $3 \mathrm{D}$ source reconstruction of the P300 component was largely confined to the somatosensory cortex and premotor cortices (Romo et al., 1993; Pleger et al., 2006). None of the observed effects extended to the prefrontal cortex, which has previously been shown to be involved in conscious tactile processing (Blankenburg et al., 2006) and graded visual consciousness (Christensen et al., 2006), and whose involvement would have been predicted by, for example, the neuronal global workspace theory (Dehaene et al., 2006). However, as we have only tested for parametric relations between ERP data and perceptual awareness ratings, it is possible that prefrontal areas are activated in a manner that does not preserve a linear relationship between their synchronous firing (approximated by ERP amplitude) and perceptual quality. For instance, recent studies on working memory suggest that stimulus properties independent of modality can be parametrically represented in the beta power of oscillations localized to the inferior frontal 
gyrus (Spitzer and Blankenburg, 2012). However, an exploratory analysis of analogous effects in our data yielded no significant results.

Furthermore, it is worth noting that the levels of early activity localized to cSI and c/iSII, while preserving an overall linear relation with parametric awareness ratings, did not distinguish between the mid-range rating bins $(25-50 \%$ and $50-75 \%)$ with 90\% confidence (Fig. 2C, left and middle). However, the activity in PMC (Fig. 2C, right), SI, and SII around the P300 component did allow for such a distinction (Del Cul et al., 2007). This pattern of results might suggest that the early sensory activity, rather than forming the neural correlate of conscious somatosensation per se, might be a prerequisite for a differentiation of perceptual quality (Aru et al., 2012; de Graaf et al., 2012), while it is the later widespread activity that encodes perceptual awareness in a more refined way.

In a complementary analysis, the late ERP components (209$400 \mathrm{~ms}$ ) were found to be susceptible to modulation by stimulus onset relative to the beginning of the stimulation window, possibly due to an increase in temporal expectation of the stimulus toward the end of the stimulation window. Behaviorally, weak tactile stimuli presented at longer latencies were associated with higher awareness ratings, consistent with previous reports that temporal expectation boosts stimulus perceivability (Rohenkohl et al., 2012). Furthermore, the latency-related differences in topographies of late ERP components could be localized to activity in the left intraparietal sulcus, which in a recent study has been directly linked to temporal orienting (Cotti et al., 2011). However, as in our experiment the distribution of stimulus onsets and the stimulus probability itself were kept constant, future studies should use an explicit manipulation of temporal expectation for a more thorough investigation of its effects on somatosensory awareness.

Finally, the predominantly feedforward activation in the longest analyzed data segment (400 ms) could be interpreted as marking a shift from awareness-related recurrent processing to movement preparation implemented in PMC (Cunnington et al., 2005; but see Boly et al., 2011). It is worth noting, however, that single-neuron recordings in the macaque somatosensory system have demonstrated that the premotor cortex and supplementary motor areas encode perceptual features of the stimulation rather than subserve mere motor preparation (Romo et al., 1993; de Lafuente and Romo, 2006). In the latter study (de Lafuente and Romo, 2006), an analysis of neuronal response latencies across the somatosensory processing stream revealed a large overlap of single-neuron responses among SI, SII, and the premotor cortex in the mid-latency range ( $\sim 50-200 \mathrm{~ms}$ poststimulus), possibly encompassing neural feedback. Later toward the response, however, only premotor and motor activity could be detected. Thus, when a large data segment is analyzed, a predominantly serial pattern might appear in the processing stream.

Together, our current findings corroborate the role of recurrent neural processing within the sensory cortices as a plausible mechanism of perceptual awareness. The strength of such recurrent processing, and the resulting activity levels in the sensory areas, could be shown to parametrically reflect subsequent perceptual awareness ratings. Finally, at least in paradigms relying on awareness ratings of simple tactile stimuli, the parametric relationship of neural activity and the subjective quality of the percept might be confined to the sensorimotor network.

\section{References}

Anderson KL, Ding M (2011) Attentional modulation of the somatosensory mu rhythm. Neuroscience 180:165-180. CrossRef Medline

Aru J, Bachmann T, Singer W, Melloni L (2012) Distilling the neural correlates of consciousness. Neurosci Biobehav Rev 36:737-746. CrossRef Medline

Auksztulewicz R, Spitzer B, Blankenburg F (2012) Recurrent neural processing and somatosensory awareness. J Neurosci 32:799-805. CrossRef Medline

Benjamini Y, Hochberg Y (1995) Controlling the false discovery rate: a practical and powerful approach to multiple testing. J R Stat Soc Ser B 57:289-300.

Blankenburg F, Ruff CC, Deichmann R, Rees G, Driver J (2006) The cutaneous rabbit illusion affects human primary sensory cortex somatotopically. PLoS Biol 4:e69. CrossRef Medline

Block N (2007) Consciousness, accessibility, and the mesh between psychology and neuroscience. Behav Brain Sci 30:481-499. CrossRef Medline

Boehler CN, Schoenfeld MA, Heinze HJ, Hopf JM (2008) Rapid recurrent processing gates awareness. Proc Natl Acad Sci U S A 105:8742-8747. CrossRef Medline

Boly M, Garrido MI, Gosseries O, Bruno MA, Boveroux P, Schnakers C, Massimini M, Litvak V, Laureys S, Friston K (2011) Preserved feedforward but impaired top-down processes in the vegetative state. Science 332:858-862. CrossRef Medline

Bressler SL, Tang W, Sylvester CM, Shulman GL, Corbetta M (2008) Topdown control of human visual cortex by frontal and parietal cortex in anticipatory visual spatial attention. J Neurosci 28:10056-10061. CrossRef Medline

Busch NA, Dubois J, VanRullen R (2009) The phase of ongoing EEG oscillations predicts visual perception. J Neurosci 29:7869-7876. CrossRef Medline

Christensen MS, Ramsøy TZ, Lund TE, Madsen KH, Rowe JB (2006) An fMRI study of the neural correlates of graded visual perception. Neuroimage 31:1711-1725. CrossRef Medline

Corbetta M, Shulman GL (2002) Control of goal-directed and stimulusdriven attention in the brain. Nat Rev Neurosci 3:201-215. CrossRef Medline

Cotti J, Rohenkohl G, Stokes M, Nobre AC, Coull JT (2011) Functionally dissociating temporal and motor components of response preparation in left intraparietal sulcus. Neuroimage 54:1221-1230. CrossRef Medline

Cunnington R, Windischberger C, Moser E (2005) Premovement activity of the pre-supplementary motor area and the readiness for action: studies of time-resolved event-related functional MRI. Hum Mov Sci 24:644-656. CrossRef Medline

David O, Kiebel SJ, Harrison LM, Mattout J, Kilner JM, Friston KJ (2006) Dynamic causal modeling of evoked responses in EEG and MEG. Neuroimage 30:1255-1272. CrossRef Medline

de Graaf TA, Hsieh PJ, Sack AT (2012) The "correlates" in neural correlates of consciousness. Neurosci Biobehav Rev 36:191-197. CrossRef Medline

Dehaene S, Changeux JP (2011) Experimental and theoretical approaches to conscious processing. Neuron 70:200-227. CrossRef Medline

Dehaene S, Changeux JP, Naccache L, Sackur J, Sergent C (2006) Conscious, preconscious, and subliminal processing: a testable taxonomy. Trends Cogn Sci 10:204-211. CrossRef Medline

Del Cul A, Baillet S, Dehaene S (2007) Brain dynamics underlying the nonlinear threshold for access to consciousness. PLoS Biol 5:e260. CrossRef Medline

de Lafuente V, Romo R (2006) Neural correlate of subjective sensory experience gradually builds up across cortical areas. Proc Natl Acad Sci U S A 103:14266-14271. CrossRef Medline

Eickhoff SB, Stephan KE, Mohlberg H, Grefkes C, Fink GR, Amunts K, Zilles K (2005) A new SPM toolbox for combining probabilistic cytoarchitectonic maps and functional imaging data. Neuroimage 25:1325-1335. CrossRef Medline

Fahrenfort JJ, Scholte HS, Lamme VAF (2008) The spatiotemporal profile of cortical processing leading up to visual perception. J Vis 8(1):12 1-12. CrossRef Medline

Garrido MI, Kilner JM, Kiebel SJ, Friston KJ (2007) Evoked brain responses are generated by feedback loops. Proc Natl Acad Sci U S A 104:2096120966. CrossRef Medline

Greenberg AS, Esterman M, Wilson D, Serences JT, Yantis S (2010) Control 
of spatial and feature-based attention in frontoparietal cortex. J Neurosci 30:14330-14339. CrossRef Medline

Haegens S, Händel BF, Jensen O (2011) Top-down controlled alpha band activity in somatosensory areas determines behavioral performance in a discrimination task. J Neurosci 31:5197-5204. CrossRef Medline

Hagiwara K, Okamoto T, Shigeto H, Ogata K, Somehara Y, Matsushita T, Kira J, Tobimatsu S (2010) Oscillatory gamma synchronization binds the primary and secondary somatosensory areas in humans. Neuroimage 51: 412-420. CrossRef Medline

Hämäläinen MS, Ilmoniemi RJ (1994) Interpreting magnetic fields of the brain: minimum norm estimates. Med Biol Eng Comput 32:35-42. CrossRef Medline

Hesselmann G, Malach R (2011) The link between fMRI-BOLD activation and perceptual awareness is "stream-invariant" in the human visual system. Cereb Cortex 21:2829-2837. CrossRef Medline

Hesselmann G, Hebart M, Malach R (2011) Differential BOLD activity associated with subjective and objective reports during "blindsight" in normal observers. J Neurosci 31:12936-12944. CrossRef Medline

Jones SR, Pritchett DL, Stufflebeam SM, Hämäläinen M, Moore CI (2007) Neural correlates of tactile detection: a combined magnetoencephalography and biophysically based computational modeling study. J Neurosci 27:10751-10764. CrossRef Medline

Kiebel SJ, Daunizeau J, Phillips C, Friston KJ (2008) Variational Bayes inversion of the equivalent current dipole model in EEG/MEG. Neuroimage 39:728-741. CrossRef Medline

Kilner JM, Kiebel SJ, Friston KJ (2005) Applications of random field theory to electrophysiology. Neurosci Lett 374:174-178. CrossRef Medline

Koivisto M, Revonsuo A (2008) The role of selective attention in visual awareness of stimulus features: electrophysiological studies. Cogn Affect Behav Neurosci 8:195-210. CrossRef Medline

Lamme VA (2006) Towards a true neural stance on consciousness. Trends Cogn Sci 10:494-501. CrossRef Medline

Linkenkaer-Hansen K, Nikulin VV, Palva S, Ilmoniemi RJ, Palva JM (2004) Prestimulus oscillations enhance psychophysical performance in humans. J Neurosci 24:10186-10190. CrossRef Medline

Litvak V, Mattout J, Kiebel S, Phillips C, Henson R, Kilner J, Barnes G, Oostenveld R, Daunizeau J, Flandin G, Penny W, Friston K (2011) EEG and MEG data analysis in SPM8. Comput Intell Neurosci 2011:852961. CrossRef Medline

Liu Y, Paradis AL, Yahia-Cherif L, Tallon-Baudry C (2012) Activity in the lateral occipital cortex between 200 and $300 \mathrm{~ms}$ distinguishes between physically identical seen and unseen stimuli. Front Hum Neurosci 6:211. CrossRef Medline

Luck SJ, Woodman GF, Vogel EK (2000) Event-related potential studies of attention. Trends Cogn Sci 4:432-440. CrossRef Medline

Mathewson KE, Gratton G, Fabiani M, Beck DM, Ro T (2009) To see or not to see: prestimulus alpha phase predicts visual awareness. J Neurosci 29: 2725-2732. CrossRef Medline

Pascual-Leone A, Walsh V (2001) Fast backprojections from the motion to the primary visual area necessary for visual awareness. Science 292: 510-512. CrossRef Medline

Penny WD, Stephan KE, Daunizeau J, Rosa MJ, Friston KJ, Schofield TM, Leff AP (2010) Comparing families of dynamic causal models. PLoS Comput Biol 6:e1000709. CrossRef Medline

Petersen SE, Posner MI (2012) The attention system of the human brain: 20 years later. Annu Rev Neurosci 35:73-89. CrossRef Medline
Pins D, Ffytche D (2003) The neural correlates of conscious vision. Cereb Cortex 13:461-474. CrossRef Medline

Pleger B, Ruff CC, Blankenburg F, Bestmann S, Wiech K, Stephan KE, Capilla A, Friston KJ, Dolan RJ (2006) Neural coding of tactile decisions in the human prefrontal cortex. J Neurosci 29:12596-12601. CrossRef Medline

Ro T, Breitmeyer B, Burton P, Singhal NS, Lane D (2003) Feedback contributions to visual awareness in human occipital cortex. Curr Biol 11:1038-1041.

Rohenkohl G, Cravo AM, Wyart V, Nobre AC (2012) Temporal expectation improves the quality of sensory information. J Neurosci 32:8424-8428. CrossRef Medline

Romo R, Ruiz S, Crespo P, Zainos A, Merchant H (1993) Representation of tactile signals in primate supplementary motor area. J Neurophysiol 70: 2690-2694. Medline

Romo R, Lemus L, de Lafuente V (2012) Sense, memory, and decisionmaking in the somatosensory cortical network. Curr Opin Neurobiol 22:914-919. CrossRef Medline

Salti M, Bar-Haim Y, Lamy D (2012) The P3 component of the ERP reflects conscious perception, not confidence. Conscious Cogn 21:961-968. CrossRef Medline

Sandberg K, Timmermans B, Overgaard M, Cleeremans A (2010) Measuring consciousness: is one measure better than the other? Conscious Cogn 19:1069-1078. CrossRef Medline

Schubert R, Blankenburg F, Lemm S, Villringer A, Curio G (2006) Now you feel it-now you don't: ERP correlates of somatosensory awareness. Psychophysiology 43:31-40. CrossRef Medline

Schubert R, Haufe S, Blankenburg F, Villringer A, Curio G (2009) Now you'll feel it, now you won't: EEG rhythms predict the effectiveness of perceptual masking. J Cogn Neurosci 21:2407-2419. CrossRef Medline

Spitzer B, Blankenburg F (2012) Supramodal parametric working memory processing in humans. J Neurosci 32:3287-3295. CrossRef Medline

Steinmetz PN, Roy A, Fitzgerald PJ, Hsiao SS, Johnson KO, Niebur E (2000) Attention modulates synchronized neuronal firing in primate somatosensory cortex. Nature 404:187-190. CrossRef Medline

Stephan KE, Penny WD, Daunizeau J, Moran RJ, Friston KJ (2009) Bayesian model selection for group studies. Neuroimage 46:1004-1017. CrossRef Medline

Stephan KE, Penny WD, Moran RJ, den Ouden HE, Daunizeau J, Friston KJ (2010) Ten simple rules for dynamic causal modeling. Neuroimage 49: 3099-3109. CrossRef Medline

Supp GG, Siegel M, Hipp JF, Engel AK (2011) Cortical hypersynchrony predicts breakdown of sensory processing during loss of consciousness. Curr Biol 21:1988-1993. CrossRef Medline

Tallon-Baudry C (2011) On the neural mechanisms subserving consciousness and attention. Front Psychol 2:397. CrossRef Medline

Thees S, Blankenburg F, Taskin B, Curio G, Villringer A (2003) Dipole source localization and fMRI of simultaneously recorded data applied to somatosensory categorization. Neuroimage 18:707-719. CrossRef Medline

Wyart V, Tallon-Baudry C (2008) A neural dissociation between visual awareness and spatial attention. J Neurosci 28:2667-2679. CrossRef Medline

Zeki S (2001) Localization and globalization in conscious vision. Annu Rev Neurosci 24:57-86. CrossRef Medline

Zhang Y, Ding M (2010) Detection of a weak somatosensory stimulus: role of the prestimulus mu rhythm and its top-down modulation. J Cogn Neurosci 22:307-322. CrossRef Medline 